\title{
Shape Determination in Lithium-Ion Battery Cathode Materials Using Electron Diffraction-Assisted Electron Tomography
}

\author{
Alpesh Khushalchand Shukla ${ }^{1,4^{*}}$, Colin Ophus ${ }^{2}$, Joan Mendoza ${ }^{3}$, Lluis Yedra ${ }^{3}$, Sonia Estrade ${ }^{3}$ and \\ Quentin Ramasse ${ }^{4}$ \\ 1. Energy Storage and Distributed Resources Division, Lawrence Berkeley National Laboratory, \\ Berkeley, California, USA. \\ 2. National Center of Electron Microscopy, Molecular Foundry, Lawrence Berkeley National \\ Laboratory, Berkeley, California, USA. \\ 3. Department of Electronics and Biomedical Engineering, University of Barcelona, Barcelona, Spain. \\ 4. SuperSTEM Laboratory, Daresbury, United Kingdom. \\ * Corresponding author: akshukla@lbl.gov
}

Commercial cathode materials used in lithium ion batteries such as lithium transition metal oxides usually consist of 5 to 10 micron-sized, spherical secondary particles. These particles consist of primary particles that are connected to each other akin to seeds in a pomegranate. These primary particles are nearly equiaxed in shape and highly faceted. Recent aberration-corrected STEM- based studies have shown that the select facets of these particles consists of a thin layer of spinel phase [1-3]. The spinel phase is formed specifically on the facets that correspond to the diffusion path of lithium ions. For example, in Li- and Mn-rich transition metal oxides (LMRTMO), they form on $\{131\}$ monoclinic facets but do not form on the stable (001) monoclinic facets. To completely understand the intercalation and phase transformation mechanisms in these materials, it is important to characterize the morphological shape of these particles.

In this study, we present results from an electron diffraction-assisted electron tomography experiment, whereby all the facets of LMRTMO particles were characterized and indexed using very few projections taken at high symmetry zone axes (Figure 1). Orientation relationship between the parent monoclinic phase and spinel phase obtained from atomic resolution HAADF images were then input to quantify the spinel surface present on the crystal. As shown in Figure 2, among all the facets that facilitate the twodimensional diffusion of lithium (essentially all facets except $(001)$ and $(00 \overline{1})$ ), a significant surface area is covered by (131) and equivalent facets that consist of a thin spinel surface and therefore their presence should not be ignored while performing phase transformation studies in cycled cathode materials.

Results from theoretical studies [4] that use Wulff theorem [5] to estimate equilibrium shapes will be compared to the experimentally obtained results, and the limitations of theoretical predictions as well as experimental results will be discussed [6].

\section{References:}

[1] AK Shukla et al., Energy \& Environmental Science 11 (2018), p. 830.

[2] S Kuppan et al., Energy Mater. 17 (2017), p. 1602010.

[3] AK Shukla et al., Nat Comm 6 (2015), p. 8711.

[4] D Kramer and G Ceder, Chem. Mater. 21 (2009), p. 3799.

[5] G Wulff, Zeitschrift für Kristallographie 34 (1901), p. 449. 
[6] Work at the Molecular Foundry was supported by the Office of Science, Office of Basic Energy Sciences, of the US Department of Energy under Contract No. DE-AC02-05CH11231. Authors sincerely thank Dr. Guoying Chen and Dr. Vince Battaglia for providing samples used in this study.
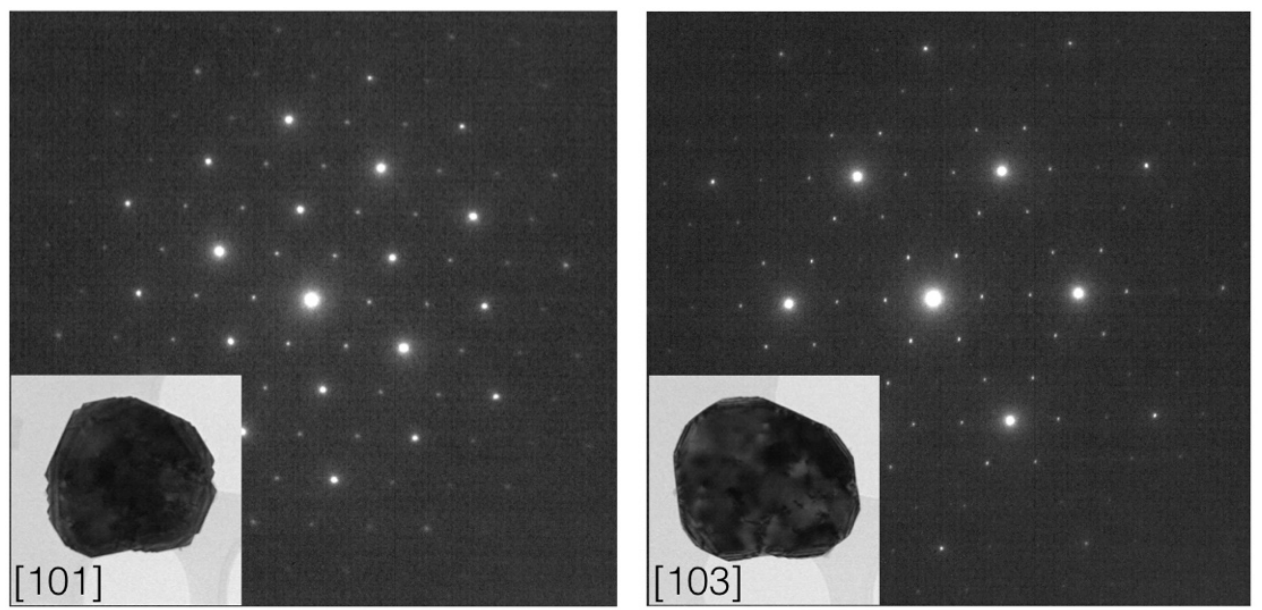

Figure 1. Diffraction patterns and corresponding bright field images taken at high symmetry zone axes., A total of eight projections were used to completely reconstruct the shape of the crystal by measuring the distance between facets and comparing them to the shapes produced by Wulff construction.

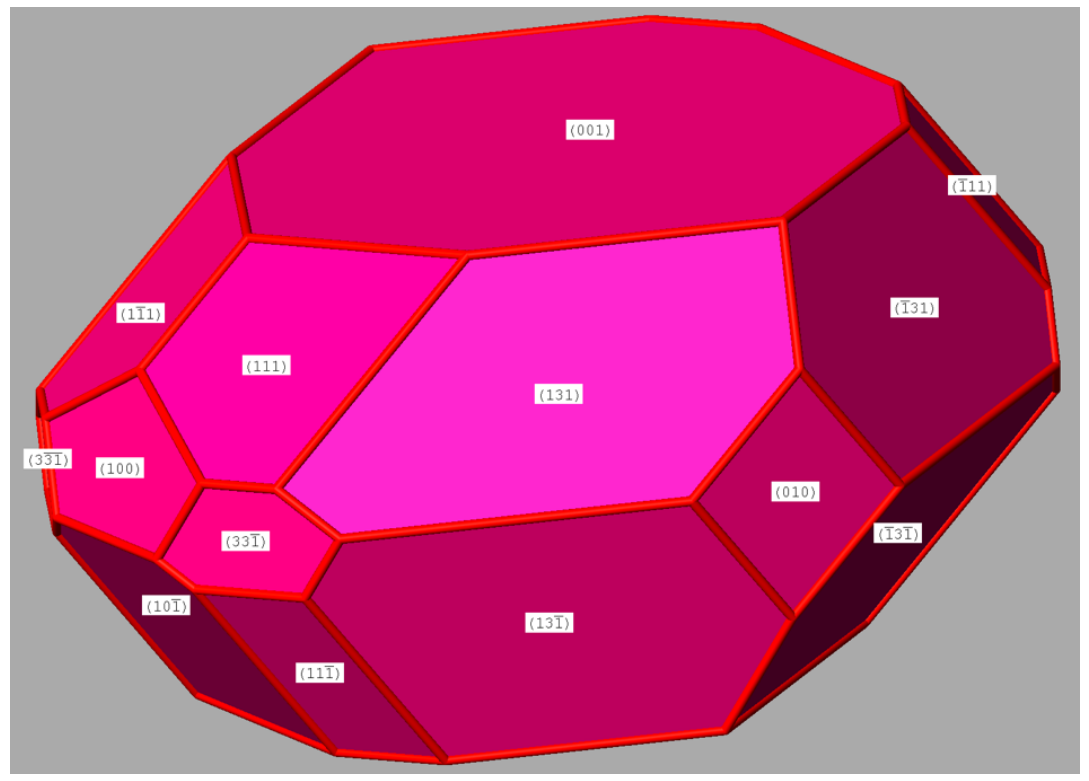

Figure 2. Model obtained using an electron diffraction-assisted electron tomography experiment for lithium and manganese-rich transition metal oxide showing different facets. (131) (highlighted) and other equivalent facets consist of a spinel surface. 